\title{
Characteristics and Surgical Outcomes of Intertrochanteric or Subtrochanteric Fractures Associated with Ipsilateral Femoral Shaft Fractures Treated with Closed Intramedullary Nailing: A Review of 31 Consecutive Cases over Four Years at a Single Institution
}

\author{
Yoon Jae Seong, MD, Jae Hoon Jang, MD*, Se Bin Jeon, MD*, Nam Hoon Moon, MD, PhD* \\ Department of Orthopaedic Surgery, Myung Eun Hospital, Busan, Korea \\ Department of Orthopaedic Surgery, Bio-Medical Research Institute, Pusan National University Hospital, Busan, Korea*
}

Purpose: To evaluate the clinical characteristics of intertrochanteric or subtrochanteric fractures associated with ipsilateral femoral shaft fractures and assess the surgical outcomes of a novel, closed intramedullary nailing surgical approach designed to minimize fixation failure.

Materials and Methods: Between May 2013 and April 2017, 31 patients with intertrochanteric or subtrochanteric fractures associated with ipsilateral femoral shaft fractures treated with closed intramedullary nailing or long proximal femoral nail antirotation (PFNA) were enrolled in this study. Preoperative data included age, sex, injury severity score, body mass index, location of shaft fracture, injury mechanism, accompanying traumatic injury, walking ability before injury, and surgical timing. Perioperative outcomes, including follow-up period, types of intramedullary nails, number of blocking screws used, operation time, and blood loss were assessed. Radiologic outcomes, including union rate, time from surgery to union, and femoral shortening, and clinical outcomes, including hip flexion, walking ability, and Harris hip score were also evaluated.

Results: A total of 29 unions (93.5\%) were achieved. The time to union was 16.8 months (range, 11-25 months) for hip fractures (15.7 weeks for intertrochanteric fractures and 21.7 weeks for subtrochanteric fractures) and 22.8 months for femoral shaft fractures. There were no significant differences in surgical outcomes between the two groups except for type of intramedullary nail.

Conclusion: Closed intramedullary nailing in the treatment of intertrochanteric or subtrochanteric fractures

Submitted: May 6, 2019 1st revision: June 17, 2019

2nd revision: July 7, 2019 Final acceptance: July 8, 2019

Address reprint request to

Nam Hoon Moon, MD, PhD

(https://orcid.org/0000-0001-9975-0992)

Department of Orthopaedic Surgery, Bio-Medical Research

Institute, Pusan National University Hospital, 179 Gudeok-ro,

Seo-gu, Busan 49241, Korea

TEL: +82-51-240-7248 FAX: +82-51-247-8395

E-mail: namhoonmoondgmail.com
This is an Open Access article distributed under the terms of the Creative Commons Attribution Non-Commercial License (http://creativecommons. org/licenses/by-nc/4.0) which permits unrestricted non-commercial use, distribution, and reproduction in any medium, provided the original work is properly cited. 
Yoon Jae Seong et al. Intertrochanteric or Subtrochanteric Fractures Associated with Ipsilateral Femoral Shaft Fractures

associated with ipsilateral femoral shaft fractures may be a good surgical option. However, fixation of femoral shaft fractures might not be sufficient depending on the implant design.

Key Words: Ipsilateral femoral fracture, Femoral shaft fracture, Intramedullary nailing, Intertrochanteric fracture, Subtrochanteric fracture

\section{INTRODUCTION}

Intertrochanteric or subtrochanteric fractures associated with ipsilateral femoral shaft fractures are rare and related to high-energy trauma (e.g., falls from height, motor vehicle accidents, pedestrian traffic accidents) $)^{1-5}$. Although there are a few reports summarizing surgical outcomes of intertrochanteric or subtrochanteric fractures associated with ipsilateral femoral shaft fractures, surgical treatment still has been known to be challenging and outcomes are unpredictable due to the complexity and diversity of the fracture characteristics. A meta-analysis reported that plate combinations for the treatment of this type of fracture were associated with a high incidence of infection and nonunion, and unlocked intramedullary nail fixation was also complicated by rotary malalignment and shortening ${ }^{6,7}$.

The advantages of closed approaches to treat femoral fractures with intramedullary nails or long proximal femoral nail antirotation (PFNA; Depuy Synthes, Oberdorf, Switzerland) have been well-documented in recent studies ${ }^{8-10}$. However, there are few clinical studies characterizing the surgical outcomes of intertrochanteric or subtrochanteric fractures associated with ipsilateral femoral shaft fracture treated with closed intramedullary nailing. Thus, the aim of the present study was evaluate the clinical characteristics of intertrochanteric or subtrochanteric fractures associated with ipsilateral femoral shaft fracture and assess the surgical outcomes of our novel surgical approach to closed intramedullary nailing designed to minimize fixation failure.

\section{MATERIALS AND METHODS}

\section{Study Population and Definitions}

This study followed Declaration of Helsinki guidelines and was approved by Pusan National University Hospital institutional review board (H1905-022-079). This multicenter, retrospective observational study was conducted in a single tertiary Pusan National University Hospital with a level
I trauma center. Between May 2013 and April 2017, 31 consecutive patients with intertrochanteric or subtrochanteric fractures associated with ipsilateral femoral shaft fractures treated with closed intramedullary nailing or long PFNA were enrolled in this study. There were 23 male and 8 female with a mean age of 46.4 years (range, 21-73 years). All 31 cases involved femoral shaft fractures concurrent with intertrochanteric $(n=13)$ or subtrochanteric $(n=18)$ fractures, respectively. A peritrochanteric fracture was defined as an extra-capsular hip fracture involving the trochanter and frequent extension into the subtrochanteric region. The subtrochanteric region was regarded as the area from the lesser trochanter to $5 \mathrm{~cm}$ distal according to Fielding's classification, while the distal portion was considered the femoral shaft ${ }^{11}$. The femoral shaft (from the lower border of the lesser trochanter to the upper border of the condyle) was evenly divided into five segments, and the fourth segment was defined as the infra-isthmus region $^{12,13}$. We excluded open fractures, pathologic fractures, and patients with follow-up of less than one year.

\section{Surgical Technique and Postoperative Care}

All operations were performed with the patient in the supine position on a radiolucent fracture table with proper traction under fluoroscopy. The patella was positioned neutrally or slightly medially, a step which we consider the most important prior to starting the surgery to help ensure proper femoral alignment. Since it was difficult to reduce the displaced proximal fragments using only simple traction in a majority of our cases, a minimally invasive reduction technique was performed using various surgical instruments as shown in Fig. 1 (e.g., long Kelly forcep, Hoffman retractor, Schanz screw, bone hook, and collinear clamp). For the closed reduction of the femoral shaft fracture, a Steinmann pin or Schantz screw was used when manual reduction or closed reduction with the F-tool (Depuy Synthes) failed. Closed intramedullary nailing was performed according to the manufacturer's guideline and a conventional fracture fixation approach. 


\section{Hip \& Pelvis}

Hip Pelvis 31(4): 190-199, 2019

The PFNA was preferred for peritrochanteric fractures with intertrochanteric components to increase the fixation power of proximal fragments and achieve compression of the fracture site by sliding between the helical blade and nail. Because more options were available for the Expert Asian Femoral Nail (A2FN; Depuy Synthes) than PFNA, Expert A2FN was preferred for subtrochanteric fractures without intertrochanteric components to maximize peri-fracture site stability and the distal portion of the femoral shaft (Fig. 2).

After insertion of the intramedullary nail, recon screws or a helical blade were inserted according to the type of intramedullary nail. Reduction of the proximal fragment should be maintained by the various surgical methods mentioned above until the nails and/or screws/helical blade are inserted. When the fracture site on the femoral shaft is located distally to the isthmus of the femur (infraisthmic type), careful observation using fluoroscopy is needed to confirm the displacement or angulation of the fracture, an event which may cause significant instability of the distal portion of the femur. Since the distal part of the femur is most commonly displaced posteriorly, it can be reduced by lifting the femur using the Steinmann pin and fixation of blocking screws. Blocking screws can be similarly inserted when a coronal displacement of the fracture is observed, an approach which may improve the stability of femoral shaft fracture. Also, an additional blocking screw was routinely inserted when a long PFNA was used in infra-isthmic type fractures without accurate reduction as we believe that two distal locking screws of long PFNA are insufficient to provide the strength necessary in patients with femoral shaft fractures located distally to the isthmus (Fig. 3).

All patients were encouraged to perform quadricepsstrengthening and straight-leg-raising exercises postoperatively. After removal of drains at 48 hours postoperatively, passive knee and hip range of motion (ROM) exercises were initiated. Partial weight bearing using crutches was allowed, as tolerated, for the first six weeks, and weight bearing was gradually increased based on evidence of callus formation on follow-up radiography.

\section{Clinical and Radiological Assessment}

All patients were classified into the two groups according to the type of hip fracture. Preoperative characteristics
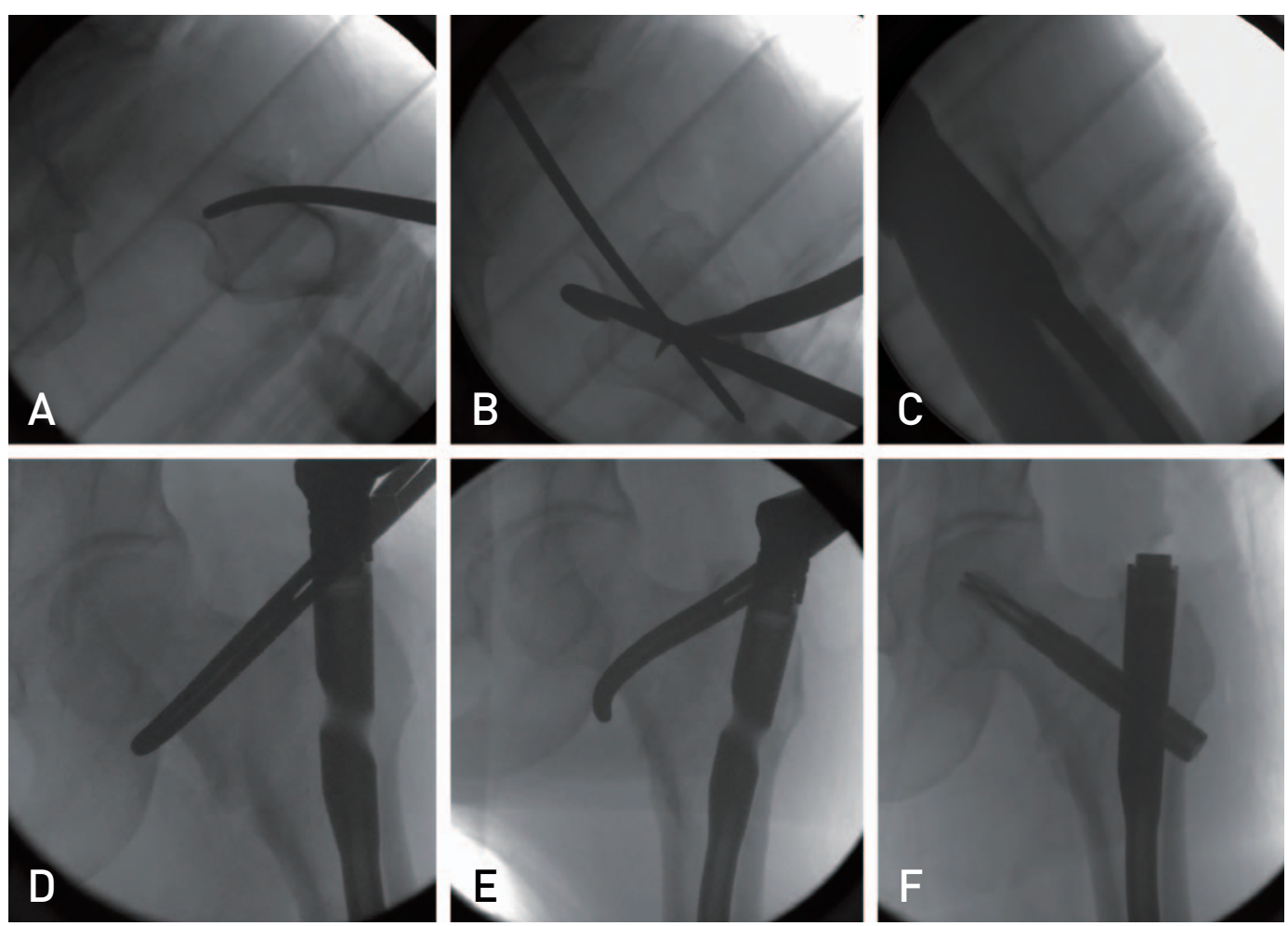

Fig. 1. The minimally invasive reduction technique designed for proximal fragments of a peritrochanteric fracture. (A-C) The reduction of flexion and external rotation deformity of the proximal fragment using long Kelly forcep and bone hook. (D-F) The reduction of medially displaced proximal fragment of intertrochanteric fracture using a long Kelly forcep. 


\section{Hip \& Pelvis}

Yoon Jae Seong et al. Intertrochanteric or Subtrochanteric Fractures Associated with Ipsilateral Femoral Shaft Fractures
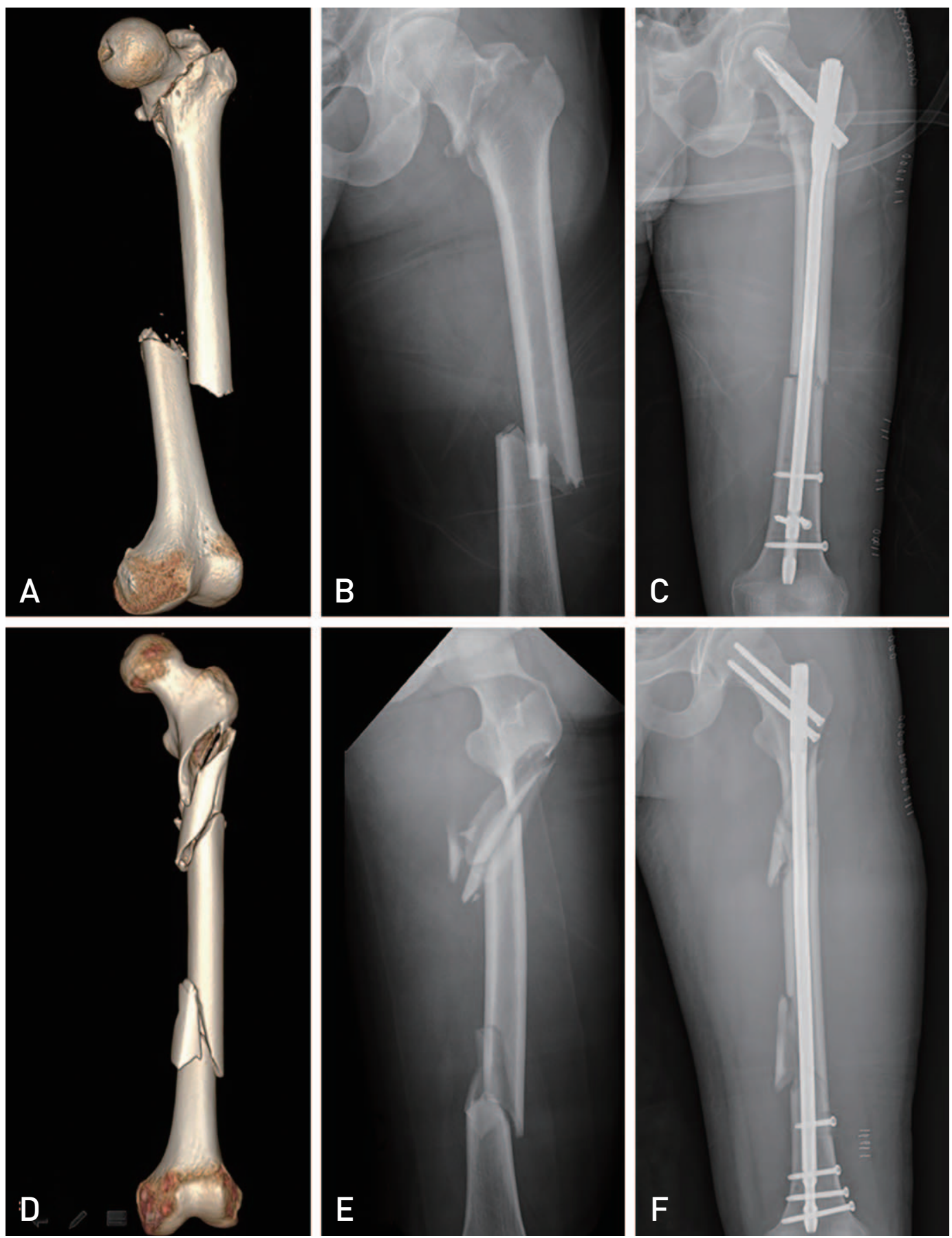

Fig. 2. (A-C) Ipsilateral intertrochanteric and femoral shaft fracture treated with long proximal femoral nail antirotation and two blocking screws. (D-F) Ipsilateral subtrochanteric and femoral shaft fracture treated with A2FN and one blocking screw.

(i.e., age, sex, injury severity score [ISS], body mass index [BMI], location of shaft fracture, injury mechanism, accompanying traumatic injuries, walking ability before injury, time from injury to surgery) were assessed. Postoperative data included operation time, types of intramedullary nails, number of blocking screws used, blood loss, and follow-up period. Union rate, time from surgery to union, and femoral shortening were also evaluated for radiologic outcome comparisons. Clinical outcomes (e.g., range of hip flexion, walking ability, Harris hip score at the last follow-up) were evaluated.

Two orthopedic surgeons confirmed fracture union (i.e., full painless weight-bearing with a bridging callus across at least three cortices on anteroposteriaor and lateral 


\section{Hip \& Pelvis}

Hip Pelvis 31(4): 190-199, 2019
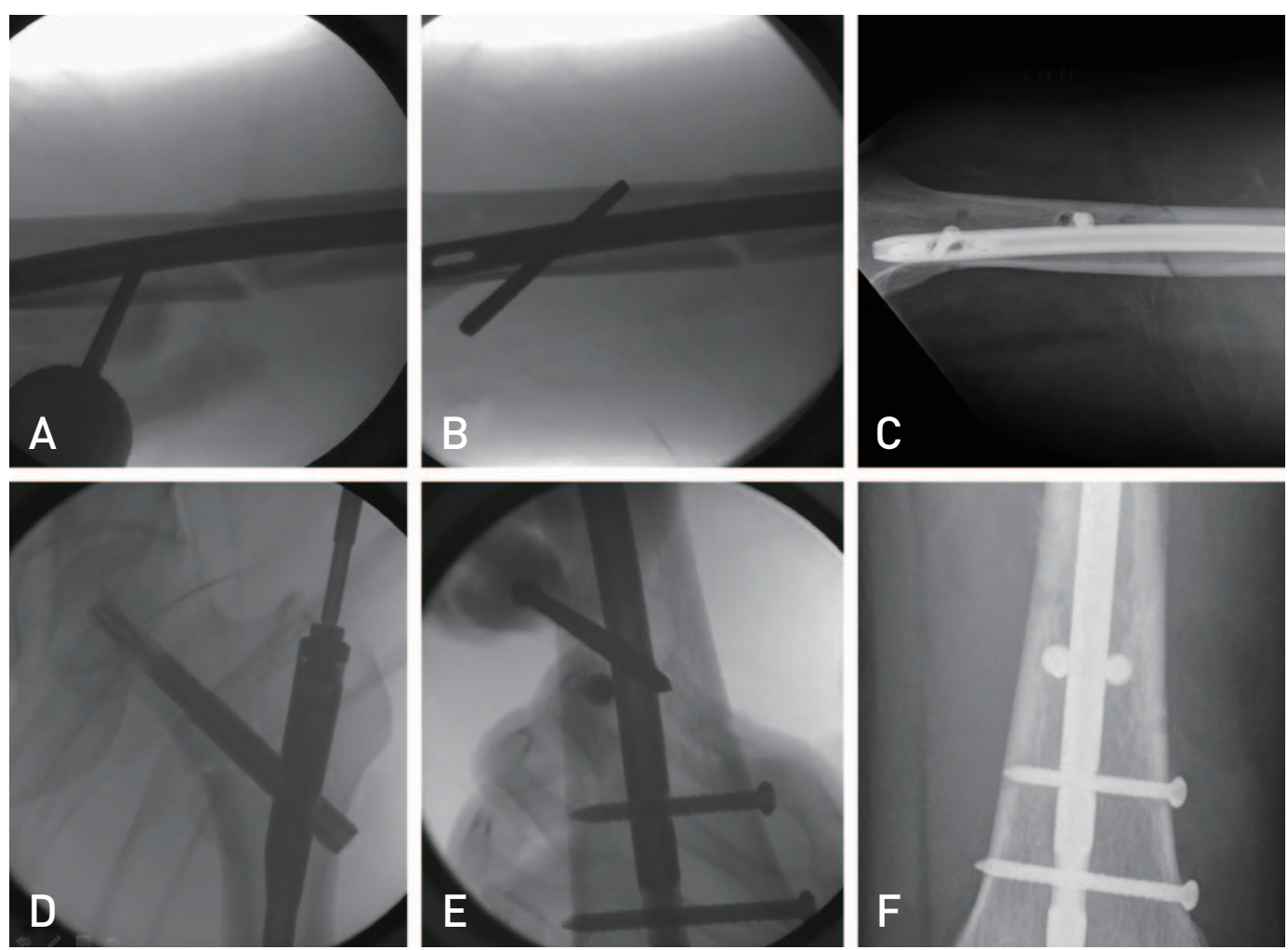

Fig. 3. (A-C) The distal portion of the femoral shaft fracture displaced posteriorly was reduced by lifting the femur using the Steinmann pin and fixation of the blocking screws. (D-F) The blocking screws were routinely inserted when the long proximal femoral nail antirotation was used in infra-isthmic type fractures.

views of the femur). Orthoroentgenography at the 1-year follow-up was used to measure shortening of the femur. Walking ability was graded from 0 to 9 using the mobility score published by Parker and Palmer, which reflects the sum of the ability to walk indoors and outdoors and to participate in social activities ${ }^{14,15}$.

\section{RESULTS}

Preoperative characteristics of all patients in the present study are provided in Table 1 . The mean ISS was 22.4 (range, 9-43) and the mean BMI was $23.3 \mathrm{~kg} / \mathrm{m}^{2}$ (range, $\left.19.1-29.0 \mathrm{~kg} / \mathrm{m}^{2}\right)$. There were 13 cases $(41.9 \%)$ of infraisthmic type fractures. Injury mechanisms included falls from a height $(\mathrm{n}=9 ; 29.0 \%)$, pedestrian traffic accidents (TA; $n=9 ; 29.0 \%)$, pedestrian TA $(n=4 ; 12.9 \%)$, and motorcycle TA $(n=9 ; 29.0 \%)$. Accompanying traumatic injuries included brain injuries $(\mathrm{n}=9 ; 29.0 \%)$, facial injuries $(\mathrm{n}=8 ; 25.8 \%)$, chest injuries $(\mathrm{n}=8 ; 25.8 \%)$, and abdominal injuries $(\mathrm{n}=6 ; 19.4 \%)$. The mean walking ability before injury was 8.6 (range, 7-9) and the mean time from injury to surgery was 4.7 days (range, $0-10$ days). There was no significant difference in preoperative demographics between the two groups except for BMI.

Surgical outcomes of all patients are presented in Table 2. The mean follow-up period was 20.1 months (range, 12-48 months). Long PFNA was used in all patients with intertrochanteric and femoral shaft fractures and in three patients with subtrochanteric and femoral shaft fractures. The mean operative time and blood loss were 168.1 minutes (range, 100-245 minutes) and $461.3 \mathrm{~mL}$, respectively. Twelve patients $(38.7 \%)$ required more than one blocking screw. For femoral shaft fractures, a total of 29 unions (93.5\%) were achieved; the remaining two cases were classified as non-union. The time to union was 16.2 weeks (range, 1125 weeks) for hip fractures (15.7 weeks for intertrochanteric fractures and 21.7 weeks for subtrochanteric fractures) and 22.8 weeks (range, 12-52 weeks) for femoral shaft fractures. The mean shortening and hip flexion at the last follow-up were $0.66 \mathrm{~cm}$ (range, $0-2.5 \mathrm{~cm}$ ) and $117.6^{\circ}$ (range, $100-120^{\circ}$ ), respectively. The mean walking ability and Harris hip score at the last follow-up were 8.4 (range, 7-9) and 90.7 (range, 73-100), respectively. There were no significant differences in surgical outcomes between the two groups except for type of intramedullary nail. 


\section{Hip \& Pelvis}

Yoon Jae Seong et al. Intertrochanteric or Subtrochanteric Fractures Associated with Ipsilateral Femoral Shaft Fractures

Table 1. Preoperative Details of 31 Ipsilateral Peritrochatneric and Femoral Shaft Fractures Treated by Closed Intramedullary Nailing

\begin{tabular}{|c|c|c|c|c|}
\hline Variable & Total & $\begin{array}{l}\text { Intertrochanteric } \\
\text { and shaft }\end{array}$ & $\begin{array}{c}\text { Subtrochanteric } \\
\text { and shaft }\end{array}$ & $P$-value \\
\hline No. of patient & 31 & 13 & 18 & \\
\hline Age at surgery (yr) & $46.4 \pm 15.7(21-73)$ & $44.1 \pm 13.8(24-67)$ & $48.5 \pm 17.1(21-73)$ & 0.449 \\
\hline Sex (male) & $23(74.2)$ & $9(69.2)$ & $14(77.8)$ & 0.592 \\
\hline Injury severity score & $22.4 \pm 9.9(9-43)$ & $25.2 \pm 11.4(9-43)$ & $20.4 \pm 8.5(9-34)$ & 0.184 \\
\hline Body mass index $\left(\mathrm{kg} / \mathrm{m}^{2}\right)$ & $23.3 \pm 2.6(19.1-29.0)$ & $24.6 \pm 2.8(19.5-29.0)$ & $22.4 \pm 2.2(19.1-26.8)$ & 0.020 \\
\hline \multicolumn{5}{|l|}{ Location of shaft fracture } \\
\hline Isthmic & $18(58.1)$ & 8 (61.5) & $10(55.6)$ & \\
\hline Infra-isthmic & 13 (41.9) & $5(38.5)$ & 8 (44.4) & 0.739 \\
\hline \multicolumn{5}{|l|}{ Injury mechanism } \\
\hline Fall from a height & $9(29.0)$ & $3(16.7)$ & $6(46.2)$ & 0.297 \\
\hline Driver's TA & $9(29.0)$ & 6 (33.3) & $3(23.1)$ & 0.297 \\
\hline Pedestrian TA & $4(12.9)$ & $4(22.2)$ & $0(0)$ & 0.297 \\
\hline Motorcycle TA & $9(29.0)$ & $5(27.8)$ & $4(30.8)$ & 0.297 \\
\hline \multicolumn{5}{|l|}{ Accompanying traumatic injury } \\
\hline Brain injury & $9(29.0)$ & $4(30.8)$ & $5(27.8)$ & 0.856 \\
\hline Face injury & $8(25.8)$ & $4(30.8)$ & $4(22.2)$ & 0.592 \\
\hline Chest injury & $8(25.8)$ & $2(15.4)$ & 6 (33.3) & 0.260 \\
\hline Abdominal injury & $6(19.4)$ & $2(15.4)$ & $4(22.2)$ & 0.634 \\
\hline Walking ability before injury & $8.6 \pm 0.6(7-9)$ & $8.8 \pm 0.4(8-9)$ & $8.6 \pm 0.6(7-9)$ & 0.294 \\
\hline $\begin{array}{l}\text { Surgical timing (time from } \\
\text { injury to surgery) (day) }\end{array}$ & $4.7 \pm 2.6(0-10)$ & $5.1 \pm 2.4(0-10)$ & $4.5 \pm 2.7(0-10)$ & 0.508 \\
\hline
\end{tabular}

Values are presented as number only, mean \pm standard deviation (range), or number (\%).

TA: traffic accident.

Table 2. Surgical Outcomes of 31 Ipsilateral Peritrochanteric and Femoral Shaft Fractures Treated by Closed Intramedullary Nailing

\begin{tabular}{|c|c|c|c|c|}
\hline Variable & Total $(n=31)$ & $\begin{array}{l}\text { Intertrochanteric } \\
\text { and shaft }(n=13)\end{array}$ & $\begin{array}{l}\text { Subtrochanteric } \\
\text { and shaft }(n=18)\end{array}$ & $P$-value \\
\hline Follow-up period (mo) & $20.1 \pm 8.9(12-48)$ & $18.3 \pm 5.4(12-30)$ & $21.3 \pm 10.7(12-48)$ & 0.356 \\
\hline \multicolumn{5}{|l|}{ Type of intramedullary nail } \\
\hline Expert A2FN & 18 & 0 & 15 & $<0.001$ \\
\hline Long PFNA & 13 & 13 & 3 & \\
\hline \multicolumn{5}{|l|}{ No. of blocking screws used } \\
\hline 0 & 19 (61.3) & $6(46.2)$ & $13(72.2)$ & 0.299 \\
\hline 1 & 3 (9.7) & $2(15.4)$ & $1(5.6)$ & 0.299 \\
\hline 2 & $8(25.8)$ & 5 (38.5) & $3(16.7)$ & 0.299 \\
\hline 3 & $1(3.2)$ & $0(0)$ & $1(5.6)$ & 0.299 \\
\hline Operative time (min) & $168.1 \pm 43.8(100-245)$ & $164.3 \pm 51.1(100-245)$ & $170.8 \pm 39.0(100-225)$ & 0.686 \\
\hline Blood loss (mL) & $461.3 \pm 216.7(200-1,200)$ & $442.3 \pm 135.2(200-600)$ & $475.0 \pm 263.6(200-1,200)$ & 0.686 \\
\hline Union & $29(93.5)$ & $12(92.3)$ & $17(94.4)$ & 0.751 \\
\hline \multicolumn{5}{|l|}{ Time to union (wk) } \\
\hline Hip fracture & $16.8 \pm 4.8(11-25)$ & $15.7 \pm 4.2(12-20)$ & $21.7 \pm 7.7(12-25)$ & 0.290 \\
\hline Shaft fracture & $22.8 \pm 11.0(12-52)$ & $21.7 \pm 7.7(12-40)$ & $23.6 \pm 13.0(12-52)$ & 0.273 \\
\hline Shortening $(\mathrm{cm})$ & $0.66 \pm 0.78(0-2.5)$ & $0.65 \pm 0.72(0-2.5)$ & $0.67 \pm 0.84(0-2.5)$ & 0.965 \\
\hline Hip flexion $\left({ }^{\circ}\right)$ & $117.6 \pm 5.3(100-120)$ & $117.7 \pm 4.4(100-120)$ & $117.5 \pm 6.0(100-120)$ & 0.923 \\
\hline $\begin{array}{l}\text { Walking ability at the last } \\
\text { follow-up }\end{array}$ & $8.4 \pm 0.8(7-9)$ & $8.7 \pm 0.6(7-9)$ & $8.2 \pm 0.9(7-9)$ & 0.111 \\
\hline HHS at the last follow-up & $90.7 \pm 9.7(73-100)$ & $93.8 \pm 7.9(73-100)$ & $88.4 \pm 10.5(73-100)$ & 0.135 \\
\hline
\end{tabular}

Values are presented as mean \pm standard deviation (range), number only, or number (\%).

HHS: Harris hip score. 


\section{DISCUSSION}

This single-center retrospective cohort study of 31 patients with intertrochanteric or subtrochanteric fractures associated with ipsilateral femoral shaft fractures: i) evaluated relevant patient characteristics, ii) assessed the surgical outcomes of a novel surgical technique designed by the authors (i.e., use of closed intramedullary nailing). Bone union was obtained in 29 of 31 patients (93.5\%) included in the present study; two cases of non-union were identified for femoral shaft fractures. The mean time to union from the surgery was 16.8 weeks (11-25 weeks) for hip fractures and 22.8 months in femoral shaft fractures.

Intertrochanteric or subtrochanteric fractures associated with ipsilateral femoral shaft fracture are associated with high-energy trauma. Furthermore, the injury mechanism of this fracture is known to be associated with the transmission of longitudinal forces proximally on a flexed femur toward the hip joint, resulting in simultaneous hip and femoral shaft fractures ${ }^{1-3)}$. In the present study, all causes of injury were high energy trauma with an average ISS of 22.4. Previous studies on hip and femoral shaft fractures have reported various fixation techniques and surgical outcomes since the first report by Delaney and Street ${ }^{16)}$ in 1953. Peskun et al. ${ }^{2}$ reported five cases of delayed union (two with dynamic hip screws and three with reconstructive nails) in a study of 26 patients with intertrochanteric or subtrochanteric fracture associated with ipsilateral femoral shaft fractures who underwent surgical treatment. Wang et al. ${ }^{17)}$ reported nonunion of two femoral shaft fractures with dynamic hip screws and one with long PFNA in their study of 23 cases of intertrochanteric or subtrochanteric fractures associated with ipsilateral femoral shaft fractures. They suggested that long PFNA could be a better choice for the treatment of these complex fractures, with several key advantages (e.g., minimal exposure, reduced perioperative blood loss, successful biological fixation of both fractures with a single implant).

With the development of recent surgical techniques, including the minimally invasive reduction technique, and improved quality of fixation devices, good surgical outcomes of hip fracture have been reported. In this study, we report a $100 \%$ union rate in hip fractures, including intertrochanteric and subtrochanteric fractures. It is wellknown that high transmitted stress is mostly concentrated on the subtrochanteric area, a region constituted mainly of thick cortical bone with poor blood supply. For these reasons, subtrochanteric fractures may have a relatively higher risk of nonunion compared with other types of hip fractures. However, many recent studies have reported high union rates of subtrochanteric fracture after intramedullary nailing with good coronal alignment based on a biologicalreduction technique. In addition, we believe that these fractures may distribute the energy impact at either site and protect each other from extreme individual damage like ipsilateral femoral neck and shaft fracture; this may be related to high union rates in hip fractures.

Previous studies have highlighted several key points regarding surgical procedures for better surgical outcomes of ipsilateral hip and shaft fractures ${ }^{1,18}$. However, most of the surgical recommendations in those studies have been limited to general guidelines of closed intramedullary nailing that minimize soft tissue damage around the fracture site and maintain good alignment. In addition, there is a lack of detailed analysis of the cause for non-union in the femoral shaft and approaches for overcoming this challenge. Based on our experience, we believe that the surgical technique is as important as the choice of implant in the treatment of intertrochanteric or subtrochanteric fractures associated with ipsilateral femoral shaft fractures. This belief is rooted in the hypothesis that unlike a fragility hip fracture, acceptable reduction of a proximal fragment with closed technique can be challenging in the peritrochanteric fracture due to high-energy injury ${ }^{19}$. Furthermore, intramedullary nailing may not provide sufficient stability in infra-isthmic type femoral shaft fractures or in cases of significant comminution around the isthmus of the femoral shaft, thus leading to an increased risk of fracture instability and subsequent hypertrophic nonunion. In particular, since long PFNA was originally designed for the surgical treatment of intertrochanteric fractures, there are no additional implant options (e.g., different diameters and/or lengths) for fixation of femoral shaft fractures. Thus, long PFNA may not be of sufficient length or diameter for fixation of the distal fragment of intertrochanteric or subtrochanteric fractures associated with ipsilateral femoral shaft fractures. Additionally, long PFNA allows for only two distal locking screws in the same direction, which cannot provide sufficient fixation for the distal fragment in infra-isthmic type femoral shaft fractures. In particular, when anatomical reduction with complete cortical opposition could not be achieved in the fracture site, significant instability of femoral shaft fractures can cause delayed union or non-union. In the present study, one case of non-union occurred in an infra-isthmic type femoral shaft fracture, and another case of non-union had severe comminution around the isthmus 


\section{Hip \& Pelvis}

Yoon Jae Seong et al. Intertrochanteric or Subtrochanteric Fractures Associated with Ipsilateral Femoral Shaft Fractures

of the femoral shaft; both cases were treated with long PFNA. Since they showed typical characteristic of hypertrophic non-union with abundant callus formation, we believe that these non-unions were related to the insufficient stability of the fracture site (Fig. 4). Thus, we suggest that long PFNA may not provide sufficiency stability to the distal fracture site due to limited implant options (e.g., length and diameter) and the importance of
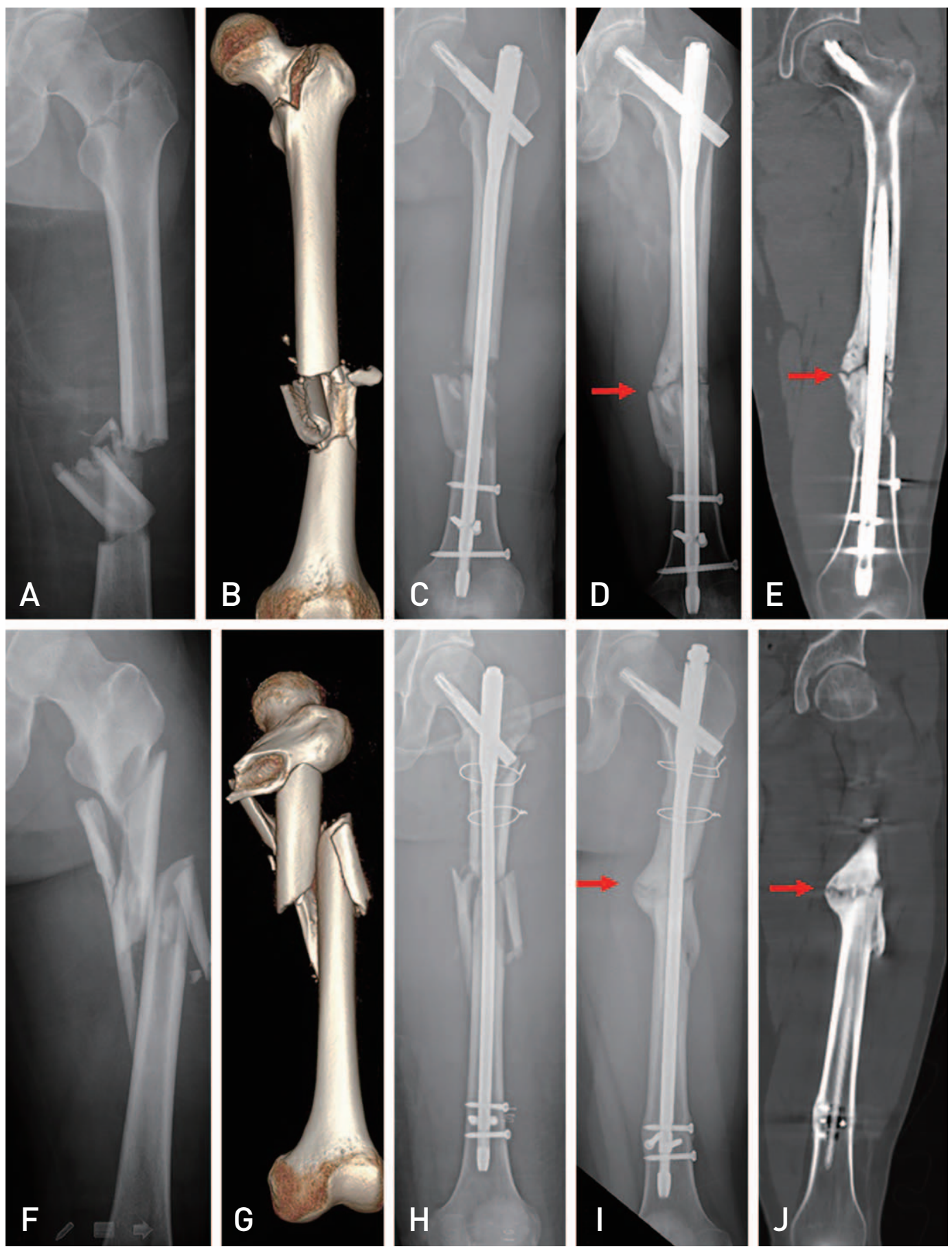

Fig. 4. (A, B) A 27-year-old male patient with an ipsilateral intertrochanteric and femoral shaft fracture. (C) Postoperative radiography following fixation with proximal femoral nail antirotation (PFNA) and three blocking screws. (D, E) Hypertrophic non-union was identified in the femoral shaft fracture (arrows). (F, G) A 24-year-old male patient with an ipsilateral subtrochanteric and femoral shaft fracture. (H) Postoperative radiography following fixation with long PFNA, percutaneous wire, and two blocking screws. (I, J) Hypertrophic non-union was identified in the femoral shaft fracture (arrows). 
stability augmentation using blocking screw might be greater when long PFNA is used.

The blocking screw technique may help overcome the problems in surgical treatment of intertrochanteric or subtrochanteric fractures associated with ipsilateral femoral shaft fractures ${ }^{20-22)}$. Hannah et al. ${ }^{21)}$ reported surgical techniques for the precise placement of poller screws with intramedullary nailing of metaphyseal fractures of the femur. They suggested that the use of a 'poller or blocking screw' may aid in satisfactory fracture reduction and biomechanical stability. We applied this blocking screw technique in 12 cases for accurate reduction and stability augmentation in patients with infra-isthmic type femoral shaft fractures or femoral shaft fractures with severe comminution around the isthmus. However, the two cases of hypertrophic non-union identified in the present study indicate that this technique may also have limitations regarding fixation stability. Thus, we recommend that design modification or diversification of the length and diameter of intramedullary nailing for distal fixation is considered by the manufacturer.

There are limitations to the present study including a small cohort size, retrospective nature of the analysis, and lack of statistical verification. Since intertrochanteric or subtrochanteric fractures associated with ipsilateral femoral shaft fractures are rare, it is difficult to collect sufficient study samples to conduct a risk-factor analysis. In particular, we were unable to conduct a statistical analysis of potential risk factors of non-union in these fractures and the association between surgical details including nail diameter, reduction quality, and nail length. Although two cases of nonunion were identified in infra-isthmic type fractures treated with long PFNA, we were unable to identify any statistical evidence for the effect of level of fracture in the shaft or implant used in the present study on surgical outcomes. Thus, we believe that further studies with larger sample sizes are needed to identify risk factors of non-union and ways to improve the surgical outcomes in intertrochanteric or subtrochanteric fractures associated with ipsilateral femoral shaft fractures.

\section{CONCLUSION}

Intertrochanteric or subtrochanteric fractures associated with ipsilateral femoral shaft fracture are rare forms of femoral fractures related to high energy injury. Additionally, these fractures are associated with poor outcomes (e.g., nonunion in the femoral shaft rather than the peritrochanteric area). Although closed intramedullary nailing in these fractures may be a good surgical treatment option, fixation of femoral shaft fractures might not be sufficient depending on the implant design. An assisted procedure (e.g., blocking screw technique) may help overcome this problem, however, it seems necessary to develop implants for stronger fixation of the distal fracture of intertrochanteric or subtrochanteric fracture associated with ipsilateral femoral shaft fractures.

\section{ACKNOWLEDGEMENTS}

This research was supported by a grant from Pusan National University Hospital, 2019.

\section{CONFLICT OF INTEREST}

The authors declare that there is no potential conflict of interest relevant to this article.

\section{REFERENCES}

1. Bali K, Gahlot N, Aggarwal S, Goni V. Cephalomedullary fixation for femoral neck/intertrochanteric and ipsilateral shaft fractures: surgical tips and pitfalls. Chin J Traumatol. 2013;16:40-5.

2.Peskun C, McKee M, Kreder H, Stephen D, McConnell A, Schemitsch EH. Functional outcome of ipsilateral intertrochanteric and femoral shaft fractures. J Orthop Trauma. 2008;22:102-6.

3. Casey MJ, Chapman MW. Ipsilateral concomitant fractures of the hip and femoral shaft. J Bone Joint Surg Am. 1979; 61:503-9.

4. Olsen M, Goshulak P, Crookshank MC, et al. Biomechanical testing of a 3-hole versus a 4-hole sliding hip screw in the presence of a retrograde intramedullary nail for ipsilateral intertrochanteric and femur shaft fractures. J Orthop Trauma. 2018;32:419-24.

5. McConnell A, Zdero R, Syed K, Peskun C, Schemitsch E. The biomechanics of ipsilateral intertrochanteric and femoral shaft fractures: a comparison of 5 fracture fixation techniques. J Orthop Trauma. 2008;22:517-24.

6. Alho A. Concurrent ipsilateral fractures of the hip and shaft of the femur. A systematic review of 722 cases. Ann Chir Gynaecol. 1997;86:326-36.

7. Alho A. Concurrent ipsilateral fractures of the hip and femoral shaft: a meta-analysis of 659 cases. Acta Orthop Scand. 1996;67:19-28.

8. Kammerlander C, Doshi H, Gebhard F, et al. Long-term results of the augmented PFNA: a prospective multicenter trial. Arch Orthop Trauma Surg. 2014;134:343-9.

9. Jiamton C, Boernert K, Babst R, Beeres FJP, Link BC. The nail-shaft-axis of the of proximal femoral nail antirotation (PFNA) is an important prognostic factor in the operative treatment of intertrochanteric fractures. Arch Orthop Trauma Surg. 2018;138:339-49. 


\section{Hip \& Pelvis}

Yoon Jae Seong et al. Intertrochanteric or Subtrochanteric Fractures Associated with Ipsilateral Femoral Shaft Fractures

10. Mereddy P, Kamath S, Ramakrishnan M, Malik H, Donnachie N. The AO/ASIF proximal femoral nail antirotation (PFNA): a new design for the treatment of unstable proximal femoral fractures. Injury. 2009;40:428-32.

11. Robinson CM, Houshian S, Khan LA. Trochanteric-entry long cephalomedullary nailing of subtrochanteric fractures caused by low-energy trauma. J Bone Joint Surg Am. 2005;87: 2217-26.

12. Wu CC, Shih CH. Distal femoral nonunion treated with interlocking nailing. J Trauma. 1991;31:1659-62.

13. Wu CC, Lee ZL. One-stage lengthening using a locked nailing technique for distal femoral shaft nonunions associated with shortening. J Orthop Trauma. 2004;18:75-80.

14. Shin WC, Moon NH, Jang JH, Lee HJ, Suh KT. Comparative study between biologic plating and intramedullary nailing for the treatment of subtrochanteric fractures: is biologic plating using LCP-DF superior to intramedullary nailing? Injury. 2017;48:2207-13.

15. Shin WC, Moon NH, Jang JH, Jeong JY, Suh KT. Technical note and surgical outcomes of percutaneous cable fixation in subtrochanteric fracture: a review of 51 consecutive cases over 4 years in two institutions. Injury. 2019;50:409-14.

16. Delaney WM, Street DM. Fracture of femoral shaft with fracture of neck of same femur; treatment with medullary nail for shaft and Knowles pins for neck. J Int Coll Surg. 1953;19:303-12.

17. Wang WY, Yang TF, Liu L, Pei FX, Xie LM. A comparative study of ipsilateral intertrochanteric and femoral shaft fractures treated with long proximal femoral nail antirotation or plate combinations. Orthop Surg. 2012;4:41-6.

18. Lin YB, Li RB, Xiong GS, et al. Treatment of middle-up part long-segment femoral fracture with long proximal femoral nail antirotation. Orthop Surg. 2015;7:138-45.

19. Jang JH, Ahn JM, Lee HJ, Moon NH. Surgical outcomes of biologic fixation for subtrochanteric fracture using locking compression plates. Hip Pelvis. 2017;29:68-76.

20. Eom TW, Kim JJ, Oh HK, Kim JW. Challenge to treat hypertrophic nonunion of the femoral shaft: the Poller screw augmentation technique. Eur J Orthop Surg Traumatol. 2016;26:559-63.

21. Hannah A, Aboelmagd T, Yip G, Hull P. A novel technique for accurate Poller (blocking) screw placement. Injury. 2014; 45:1011-4.

22. Özmeriç A, Alemdaroğlu KB. A more practical way to place a correct Poller (blocking) screw. Injury. 2016;47:510-1. 